loneliness and poor relationship quality have profoundly detrimental effects on health and wellbeing. The experiences of loneliness and poor relationship quality are though to a elicit a stress response, which when experienced chronically, can result in behavioural biases and negative physical and mental health outcomes. In comparison, the experience of high quality relationships can buffer the negative effects of other external stressors. To date, little is known about how the perceptions of loneliness and relationship quality within couples coping with a disability affect wellbeing, or how the partners' perceptions of loneliness and relationship quality affect the individuals' wellbeing, furthermore the directionality of effect is not well understood.

Methods In this study, we investigate the actor and partner effects, and the reciprocal effects of loneliness and relationship quality on wellbeing. The analyses are based on longitudinal dyadic data from the pro-WELL study $(n=246)$, a Swiss community survey of persons with spinal cord injury (SCI) and their partners. Data was collected over a 12 month period using telephone interviews and questionnaires. We employed mixed effects modelling to explore actor and partner effects, and used cross-lagged path analysis to explore reciprocal effects. Both of these analyses were stratified to account for the potential differential effects in persons with SCI and their caregiving partners.

Results We found loneliness to be more prevalent in persons with SCI compared to their caregiving partners. Caregiving partners were found to be more sensitive to their own, and their partners' experiences of relationship quality, as we found significant actor effects $(\beta=0.15 \mathrm{CI}: 0.04,0.26)$, and significant partner effects of relationship quality on wellbeing $(\beta=0.21$ CI:0.10, 0.32). In both persons with SCI $(\beta=-0.30$ CI: $-0.41,-0.18)$ and their caregiving $(\beta=-0.20 \mathrm{CI}:-0.31,-$ $0.10)$, we found significant actor effects of loneliness on wellbeing. Over time, loneliness demonstrated reciprocal associations with wellbeing, as did relationship quality in caregiving partners, indicating a possible cycle of positive development between reducing loneliness, improving relationship quality and enhancing wellbeing.

Conclusion The findings of our study emphasise the high prevalence of loneliness in persons with SCI and the importance of reducing loneliness and strengthening relationship quality in order to improve wellbeing in couples coping with disability.

\section{OP73 THE IMPACT OF MAJOR MENTAL ILLNESS ON RISK OF STROKE AND MYOCARDIAL INFARCTION IN PEOPLE WITH TYPE 2 DIABETES IN SCOTLAND: AN ANALYSIS OF ROUTINELY COLLECTED HEALTH DATA}

\begin{abstract}
${ }^{1,2} \mathrm{~K}$ Fleetwood*, ${ }^{1,2} \mathrm{~S}$ Wild, ${ }^{3} \mathrm{D}$ Smith, ${ }^{4} \mathrm{~K}$ Licence, ${ }^{3} \mathrm{~S}$ Mercer, ${ }^{1} \mathrm{C}$ Sudlow, ${ }^{1,2} \mathrm{C}$ Jackson. ${ }^{1}$ Usher Institute of Population Health Sciences and Informatics, University of Edinburgh, Edinburgh, UK; ${ }^{2}$ On behalf of the Scottish Diabetes Research Network Epidemiology Group; ${ }^{3}$ Institute of Health and Wellbeing, University of Glasgow, Glasgow, UK; ${ }^{4}$ Public Health and Intelligence, NHS National Services Scotland, Edinburgh, UK
\end{abstract}

10.1136/jech-2018-SSMabstracts.72

Background In Scotland, major mental illness reduces life expectancy by approximately 17 years, with cardiovascular disease (CVD) the leading cause of death. We aimed to investigate whether history of major mental illness affects CVD risk in people with type 2 diabetes mellitus (T2DM) in Scotland.
Methods We identified adults diagnosed with T2DM between 2004 and 2016 from a national diabetes register (the Scottish Care Information - Diabetes database). We ascertained history of mental illness from psychiatric and acute hospital admission records and incident/recurrent stroke or myocardial infarction (abbreviated to CVD) from acute hospital admission and mortality records. Using Cox regression analysis, we obtained hazard ratios (HRs) for CVD risk among people with a history of schizophrenia, bipolar disorder or depression, compared to those with T2DM but without a history of mental illness.

Results We included 212011 people with T2DM. Of these, 2107 (1.0\%) had schizophrenia, 1521 (0.7\%) had bipolar disorder and $5288(2.5 \%)$ had depression. People with these major mental disorders were younger at diagnosis of T2DM and had higher prevalence of smoking and history of alcohol use disorders than the comparison group. After adjusting for age, sex, area-based deprivation, hypertension and previous history of CVD, HRs (95\% confidence interval) were 1.27 $(1.05,1.53)$ for schizophrenia, $1.45(1.23,1.70)$ for bipolar disorder and $1.50(1.37,1.65)$ for depression compared to those without a history of mental illness. Additional adjustment for smoking and alcohol use disorder attenuated effect estimates to $1.12(0.93,1.36)$ for schizophrenia, 1.35 (1.15, 1.59) for bipolar disorder and $1.37(1.25,1.51)$ for depression.

Conclusion Schizophrenia, bipolar disorder and depression are associated with increased CVD risk among people with T2DM and this is partially explained by higher prevalence of smoking and alcohol use disorders in people with these conditions. This highlights the need for better CVD prevention in people with T2DM and major mental illness especially with respect to modifiable risk factors. Major strengths are that the diabetes register includes $99 \%$ of all people with diabetes in Scotland and the follow-up period is relatively long. However, our definition of mental illness was limited to hospital admission data only and so findings may not apply to people with mental illness not admitted to hospital. Future analyses will also include adjustment for psychotropic medication use.

\section{Health behaviours in young people}

\section{OP74 EXPLORING STUDENTS' PERCEPTIONS OF AND EXPERIENCES WITH ALCOHOL DURING THE TRANSITION TO UNIVERSITY AND ACROSS THE FIRST YEAR}

${ }^{1} \mathrm{AC}$ Fuller*, ${ }^{1} \mathrm{R}$ Murray, ${ }^{2} \mathrm{KM}$ Fleming, ${ }^{1} \mathrm{~L}$ Szatkowski, ${ }^{1} \mathrm{M}$ Bains. ${ }^{1}$ Epidemiology and Public Health, University of Nottingham, Nottingham, UK; ${ }^{2}$ Public Health and Policy, University of Liverpool, Liverpool, UK

\subsection{6/jech-2018-SSMabstracts.73}

Background Recent studies suggest binge drinking rates are high among university students in the UK. It has been suggested that many students arrive at university with established drinking patterns, but that freshers' week (FW) also serves as an introduction to the university drinking culture, and there are many non-drinkers who may feel excluded from this. The aim of this study was to explore the extent to which students' views of and experiences with alcohol change as they transition to university and across their first academic year.

Methods First year undergraduates entering a large UK university in September 2016 who completed an online Students' Union welfare survey in August 2016 were invited to 